\title{
Determinanten von beruflichen, akademischen und gemischten Bildungspfaden
}

\author{
Curdin Pfister \& Simone N. Tuor Sartore
}

\section{Zusammenfassung}

Zur Erreichung eines tertiären Bildungsabschlusses steht den Jugendlichen in der Schweiz eine Vielzahl an Bildungspfaden zur Auswahl. Ziel des vorliegenden Beitrages ist es zu analysieren, was die Wahl eines rein akademischen, eines rein beruflichen oder eines sogenannten gemischten, d.h. berufliche und akademische Ausbildungsinhalte umfassenden Bildungspfades determiniert. Den theoretischen Überlegungen folgend vermuten wir, dass sowohl die elterliche Bildung, als auch regionale und zeitliche Faktoren eine wichtige Rolle spielen dürften. Wir verwenden den Mikrozensus Aus- und Weiterbildung 2011, um unsere Hypothesen zu testen. Die empirischen Resultate zeigen, dass bei rein beruflichen und rein akademischen Bildungspfaden ein systematischer $\mathrm{Zu}$ sammenhang mit der elterlichen Bildung besteht, aber nicht so bei gemischten Bildungspfaden. Allerdings zeigen die Resultate ebenfalls, dass die Häufigkeit gemischter Bildungspfade über die Zeit zugenommen hat, was auf eine erhöhte Durchlässigkeit des Bildungssystems in jüngerer Zeit schliessen lässt.

\section{Abstract}

These days, youth can choose between various educational paths to obtain a tertiary educational degree. The aim of this study is to analyze the determinants of purely academic, purely vocational and so-called mixed, i.e. combinations of academic and vocational education, educational paths. Following our 
theoretical considerations, we hypothesize an influence of parental education, regional and temporal factors. We use the Swiss Adult Education Survey collected in 2011 to test our hypotheses. Our findings show that purely vocational and purely academic educational paths are related to parental education but not mixed paths. However, the share of mixed educational paths increased over time, suggesting an increased permeability of the national educational system in recent years.

\section{Résumé}

Les jeunes en Suisse peuvent choisir entre un grand nombre de formations pour obtenir un diplôme tertiaire. La présente contribution vise à identifier les déterminants du choix d'un parcours purement académique, d'un parcours purement professionnel ou d'un parcours mixte, soit un parcours composé des contenus et professionnels et académiques. Suivant les réflexions théoriques, nous supposons que la formation des parents ainsi que des facteurs régionaux et temporels sont d'importance. Nous testons nos hypothèses à l'aide du recensement Microrecensement formation de base et formation continue 2011. Les résultats empiriques montrent qu'il y a une relation systématique avec la formation des parents auprès des parcours purement professionnels et purement académiques, mais que ce n'est pas le cas auprès les parcours dits mixtes. Cependant, les résultats montrent aussi que le nombre des parcours mixtes a augmenté au fil du temps, dont on peut conclure que, récemment, le système de formation est devenu plus perméable.

\section{$1 \quad$ Einleitung}

Im Zuge des technologischen Fortschritts und des demographischen Wandels verändern sich auch die Anforderungen an das Arbeitskräftepotential. Einerseits besteht ein hoher Bedarf an (hoch)qualifizierten Arbeitskräften. Es erstaunt deshalb kaum, dass der Anteil von Personen zwischen 25 und 64 Jahren mit einem tertiären Bildungsabschluss (tertiär A oder B) in der Schweiz stetig zunimmt und derzeit bei 39\% liegt (BFS, 2013). Andererseits ändert sich auch die Nachfrage nach den verschiedenen Qualifikationen und es ist insbesondere zu vermuten, dass die Kombination von beruflichen und akademischen Ausbildungsinhalten an Bedeutung gewinnt. So haben in der Schweiz 21\% aller Personen mit einem tertiären Bildungsabschluss einen gemischten, d.h. berufliche und akademische 
Ausbildungen umfassenden Bildungspfad gewählt. ${ }^{1}$ Allerdings ist bisher kaum erforscht, wer einen gemischten und wer einen rein berufliche bzw. rein akademische Ausbildungsinhalte umfassenden Bildungspfad wählt. Dies ist Gegenstand des vorliegenden Beitrages.

Wir untersuchen diese Frage für die Schweiz, wo es eine grosse Vielfalt an Bildungspfaden gibt. So zeichnet sich das Schweizerische Bildungssystem als Ganzes, als auch das Berufsbildungssystem im Besondern, durch eine hohe Flexibilität, Vielfältigkeit und Durchlässigkeit aus (Culpepper, 2007; Barabasch, Scharnhorst, \& Kurz, 2009; Rohrer \& Trampusch, 2011). Die besondere Beschaffenheit des schweizerischen Bildungssystems ermöglicht so jugendlichen Entscheidungsträgern eine breite Palette an Bildungsmöglichkeiten.

Im Gegensatz zu vielen anderen europäischen und nicht-europäischen Ländern gibt es in der Schweiz - neben der klassischen akademischen Ausbildung - einen staatlich regulierten beruflichen Bildungszweig. Die bedeutende Anzahl von Personen, die ihren ursprünglichen Bildungspfad verlassen und akademische und berufliche Ausbildung kombinieren, dürfte dabei einerseits damit zusammenhängen, dass der Durchlässigkeit des schweizerischen Bildungssystems eine hohe Bedeutung beigemessen wird und diese entsprechend gefördert wird. Andererseits dürfte auch die frühe Selektion der jungen Menschen in den beruflichen oder akademischen Bildungszweig eine Rolle spielen. Obschon diese frühe Selektion in der politischen Diskussion oft als Nachteil aufgeführt wird, haben aber Tuor \& Backes-Gellner (2010) zeigen können, dass diese gemischten Bildungspfade hinsichtlich der Bildungsrendite mehr als konkurrenzfähig sind mit den anderen Bildungspfaden. Dies vor allem deshalb weil das schweizerische Bildungssystem recht durchlässig ist, auch wenn die Kombination von beruflichen und akademischen Ausbildungsinhalten durchaus noch mit Hürden verbunden sein mag. Backes-Gellner, Tuor, \& Wettstein (2010) finden zudem, dass die Kombination von beruflichen und akademischen Ausbildungsinhalten für Unternehmer von Vorteil ist. In der vorliegenden Studie soll vor diesem Hintergrund für die Schweiz der Frage nach den Determinanten der verschiedenen eingeschlagenen Bildungspfade nachgegangen werden.

Theoretische Überlegungen lassen vermuten, dass bei der Wahl eines Bildungspfades insbesondere sozioökonomische, familiäre und politische Faktoren eine Rolle spielen dürften. Aufgrund der uns für die Schweiz derzeit zur Verfügung stehenden Datenbasis fokussieren wir uns auf familiäre Faktoren und ana-

1 Diese Angabe bezieht sich auf eigene Berechnungen basierend auf dem Mikrozensus Aus- und Weiterbildung 2011. Detaillierte Informationen zur Definition der Bildungspfade sind im Kapitel 3.2 Definition Bildungspfade zu finden. 
lysieren den Zusammenhang zwischen dem Ausbildungsabschluss der Eltern und der Wahl eines bestimmten Bildungspfades. Zur Beantwortung dieser Frage unterscheiden wir zwischen Personen mit rein beruflichen Bildungspfaden, d.h. Personen, die ausschliesslich berufliche Ausbildungen absolviert haben, Personen mit rein akademischen Bildungspfaden, d.h. Personen, die ausschliesslich akademische Ausbildungen absolviert haben und Personen mit gemischten Bildungspfaden, d.h. Personen, die nach dem Start in einer Ausbildung auf dem beruflichen bzw. akademischen Bildungszweig in einem späteren Schritt auf den jeweils anderen Bildungszweig gewechselt haben. Hinsichtlich der elterlichen Bildungen wird neben dem Ausbildungsniveau ebenfalls der Ausbildungstyp (beruflich vs. akademisch) berücksichtigt. Wir vermuten, dass der Ausbildungsabschluss der Eltern, insbesondere für frühe Bildungsentscheidungen einen wesentlichen Einfluss auf den Bildungspfad ausübt. Mögliche Gründe hierfür sind die Erblichkeit intellektueller Fähigkeiten, die Investitionen hinsichtlich der Ausbildung und die Aktivitäten in Zusammenhang mit der Ausbildung.

Neben den familiären Faktoren werden in der vorliegenden Studie auch Sprachregion- und Kohorteneffekte kurz angeschnitten. Einerseits wird untersucht, ob sich die verschiedenen Bildungspfade und insbesondere die Häufigkeit von gemischten Bildungspfaden systematisch zwischen den verschiedenen Regionen in der Schweiz unterscheidet. So ist die Tradition der beruflichen Ausbildung in der deutschsprachigen Region deutlich stärker ausgeprägt als in den französisch- sowie italienischsprachigen Regionen. ${ }^{2}$ Interessant ist dabei die Frage, in welchen Regionen eher vom ursprünglich eingeschlagenen Bildungspfad abgewichen wird.

Andererseits analysieren wir Kohorteneffekte. Dies deshalb, weil jüngere Bildungsreformen (Einführung Berufsmaturität und Passerelle Berufsmaturität Universitäre Hochschulen) darauf abzielen die Durchlässigkeit und Flexibilität des Bildungssystems zu fördern. So können heute leistungsstarke Jugendliche ihre beruflichen Fähigkeiten mit vertieften Kenntnissen ergänzen (BBT, 2012, S. 3; Grob, Leu, \& Kirchhoff, 2007). Somit ist grundsätzlich zu erwarten, dass Kombinationen von akademischen und beruflichen Ausbildungsinhalten für Jugendliche aus jüngeren Kohorten mit geringeren Hürden verbunden sind und deshalb gemischte Bildungspfade mit höherer Wahrscheinlichkeit auftreten dürften.

2 Siehe dazu SKBF 2014. 


\section{Bildungspfade im Schweizerischen Bildungssystem}

Im Folgenden werden einige, für das Verständnis der Rekonstruktion der individuellen Bildungspfade wesentlichen, Merkmale des schweizerischen Bildungssystems beschrieben. ${ }^{3}$

Nach der obligatorischen Schule folgt die Sekundarstufe II auf der mit den gymnasialen Maturitätsschulen eine akademische und mit der beruflichen Grundbildung (Lehre) eine berufliche Ausbildung möglich ist. Ein Abschluss auf Sekundarstufe II ermöglicht sodann den direkten Einstieg ins Berufsleben. Daneben bietet diese Stufe aber auch die Möglichkeit sich auf die Ausbildungen der Tertiärstufe vorzubereiten. Die Tertiärstufe bietet ebenfalls wiederum mit den Universitären Hochschulen (inkl. ETH) eine akademische Ausbildung sowie mit den Fachhochschulen und der höheren Berufsbildung (Höhere Fachschule, eidgenössischen Berufsprüfung oder höheren Fachprüfung) eine berufliche Ausbildung an. ${ }^{4}$ In Abbildung 1 sind diese für die Definition der Bildungspfade wesentlichen Bildungsstufen vereinfacht dargestellt.

3 Einen detaillierten Einblick in das Schweizer Bildungssystem liefern beispielsweise die Bildungsberichte (SKBF 2010, 2014) der Schweizerischen Koordinationsstelle für Bildungsforschung. Weitere Informationen zum Schweizerischen Bildungssystem können ebenfalls dem ersten Kapitel dieses Sammelbandes „Thematische Einführung und Überblick" entnommen werden.

4 Die Hochschullandschaft der Schweiz beinhaltet - neben konventionellen Universitäten und Eidgenössischen Technischen Hochschulen - Fachhochschulen und Pädagogische Hochschulen. Fachhochschulen zeichnen sich durch praxisorientierte Studien und anwendungsorientierte Forschung und Entwicklung aus und bereiten auf berufliche Tätigkeiten und berufsqualifizierende Abschlüsse vor. Mit ihrem beruflichen Fokus stellen sie somit einen gleichwertigen, aber andersartigen Hochschultypus dar (SKBF 2014; Hoeckel et al. 2009).

Auf pädagogische Hochschulen, welche die Aus- und Weiterbildung von Lehrpersonen sicherstellen, wird in dieser Arbeit nicht eingegangen. Diese Schulen verlangen in der Regel eine gymnasiale Maturität, wobei auch Absolventen mit Berufsmaturität, bzw. einer Berufslehre, zu gewissen Studiengängen Zutritt haben. 


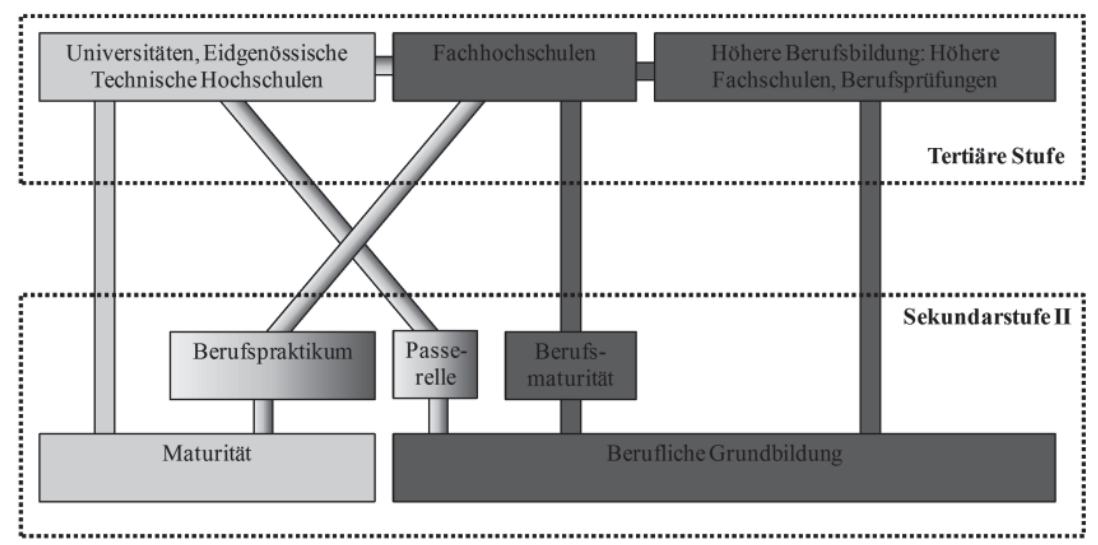

Rein Beruflich

Rein Akademisch

Gemischt

Abbildung 1 Das schweizerische Bildungssystem. Quelle: Eigene Illustration, basierend auf SKBF 2006, 2010, 2014; EDK, 1995 und dem Schweizerischen Bildungsserver (www.educa.ch).

\section{Determinanten unterschiedlicher Bildungspfade: Literatur und Hypothesen}

\subsection{Familiäre Faktoren: Ausbildungsabschluss der Eltern}

In der Literatur findet man vorwiegend drei Erklärungsansätze zum Effekt der elterlichen Bildung auf den Bildungsweg der Kinder: Erblichkeit intellektueller Fähigkeiten, Investitionen hinsichtlich der Ausbildung und Aktivitäten in Zusammenhang mit der Ausbildung. Unabhängig vom jeweiligen Kanal wird dabei ein positiver Einfluss erwartet. Zudem ist davon auszugehen, dass der Einfluss der elterlichen Bildung insbesondere bei frühen Bildungsentscheidungen zum Tragen kommt und dabei auch für die Entscheidung rein beruflicher/akademischer vs. gemischter Bildungspfad eine bedeutende Rolle spielt. Die empirische Literatur zu den Determinanten von Bildungsentscheidungen lässt tatsächlich vermuten, dass der Einfluss der elterlichen Bildung bedeutend ist, dieser jedoch für spätere Bil- 
dungsentscheidungen abnimmt. Studien, welche dabei zwischen verschiedenen Kombinationen von beruflichen und akademischen Ausbildungen unterscheiden und hinsichtlich der elterlichen Bildung Ausbildungstyp und Ausbildungsniveau berücksichtigen sind kaum vorhanden.

Erstens bringen sich Eltern im Rahmen ihrer Erziehungsaufgaben in Aktivitäten ein, die direkt oder indirekt mit der Ausbildung zusammenhängen (vgl. z.B. Leibowitz, 1974; Leibowitz, 1977; Reynolds, 1992; Fan \& Chen, 2001; Eccles \& Davies-Kean, 2005). Dies können einerseits Aktivitäten sein, die direkt mit der Ausbildung in Zusammenhang stehen wie beispielsweise die Unterstützung bei den Hausaufgaben. Andererseits kann es sich auch um Aktivitäten handeln, die intellektuelle Fähigkeiten im Allgemeinen fördern. Dabei ist zu erwarten, dass der Ausbildungsabschluss der Eltern das Ausmass und die Qualität dieser Aktivitäten massgeblich mitbestimmt.

Zweitens spielen intellektuelle Fähigkeiten bei der Ausbildung eine bedeutende Rolle. Durch die Erblichkeit von intellektuellen Fähigkeiten ist dies somit auch für den Zusammenhang zwischen der elterlichen Bildung und der Bildung der Kinder relevant. Die Diskussion dreht sich dabei insbesondere um die Frage, inwieweit die intellektuellen Fähigkeiten durch genetische Faktoren und inwieweit durch Umwelteinflüsse bestimmt werden. Plomin, DeFries, \& Fulker (1988) schlagen dabei ein theoretisches Modell vor, mit dem dies getestet werden kann. Die Resultate empirischer Studien (vgl. beispielsweise Sacerdote, 2007 oder Turkheimer, Haley, Waldron, D’Onofrio, \& Gottesman, 2003) lassen dabei keinen eindeutigen Schluss zu. Unbestritten ist aber die Tatsache, dass genetische Faktoren eine Rolle spielen bei der intergenerationalen Transmission der Ausbildung. Es ist deshalb zu erwarten, dass durch die Erblichkeit von intellektuellen Fähigkeiten die Bildung der Eltern systematisch mit der Bildung der Kinder zusammenhängt.

Drittens dürfte die Ausbildung der Eltern auch massgeblich die Investitionsentscheidung betreffend der Ausbildung der Kinder beeinflussen. Ein Grund hierfür ist, dass höhere Bildung zu durchschnittlich höheren Löhnen führt, was dann wiederum die finanziellen Ressourcen erhöht, die auch für die Bildung der Kinder verwendet werden können. Zentrale Studien in diesem Bereich sind Becker \& Tomes (1994) und Cunha \& Heckman (2007), welche sich aber auch mit anderen potentiellen Einflussfaktoren beschäftigen. Auch diesem Erklärungsansatz folgend ist zu erwarten, dass die Bildung der Eltern einen bedeutenden Einfluss auf die Bildung der Kinder ausübt. Zusammenfassend kann also festgehalten werden, dass es mehrere Kanäle gibt über welche die Ausbildung der Eltern die Ausbildung der Kinder beeinflusst, unabhängig vom jeweiligen Kanal aber ein positiver Einfluss von höherer Ausbildung zu erwarten ist. 
Die empirische Literatur zu den Determinanten von Bildungsentscheidungen lässt vermuten, dass der Einfluss der elterlichen Bildung bedeutend ist (vgl. z.B. Tansel, 2002; Connelly \& Zheng, 2003; Dustmann, 2004). Studien, welche dabei zwischen verschiedenen Bildungspfaden unterscheiden, sind sehr selten. Eine nennenswerte Ausnahme bildet Breen \& Jonsson (2000), welche für Schweden verschiedene Typen von Bildungspfaden analysieren und feststellen, dass die Bildungspfade von der sozialen Herkunft abhängen.

Neben dem Ausbildungsniveau dürfte aber insbesondere auch der Ausbildungstyp der elterlichen Bildung entscheidend sein. So dürften sich die Bildungspräferenzen der Eltern unterscheiden abhängig davon ob diese selber eine akademische oder berufliche Ausbildung absolviert haben. So zeigt Davis-Kean (2005), dass die Ausbildung der Eltern die schulischen Leistungen des Kindes über die elterlichen Erwartungen und Verhaltensweisen beeinflusst. Zudem entwickeln Kinder, die bei Eltern mit höheren Bildungsabschlüssen aufwachsen, anspruchsvollere Berufswünsche, was schliesslich auch zu einem höheren Bildungsniveau führt (Dubow, Boxer, \& Huesmann, 2009). Aufgrund der bisherigen empirischen Befunde zu Bildungspfaden ist zudem nicht davon auszugehen, dass ein bestimmter Bildungspfad systematisch bevorzugt werden sollte, werden sowohl akademische als auch berufliche Ausbildungen vom Arbeitsmarkt stark nachgefragt (Tuor \& Backes-Gellner, 2010; Backes-Gellner et al., 2010). Die erste zu testende Hypothese lautet also wie folgt:

Hypothese 1: Der Ausbildungsabschluss der Eltern hat einen signifikanten Einfluss auf den Bildungspfad der Kinder.

Dabei erwarten wir, dass Personen, deren Eltern eine akademische Ausbildung absolviert haben, mit höherer Wahrscheinlichkeit einen rein akademischen als einen rein beruflichen Bildungspfad einschlagen. Personen, deren Eltern einen beruflichen oder keinen tertiären Bildungsabschluss aufweisen, haben eine höhere Wahrscheinlichkeit einen rein beruflichen Bildungspfad einzuschlagen. Für gemischte Bildungspfade und damit die Kombination von beruflichen und akademischen Ausbildungsinhalten ist kein systematischer Zusammenhang zu einem bestimmten Ausbildungstyp elterlicher Bildung zu erwarten. Vielmehr dürften diese in allen Gruppen vertreten sein.

Gegenstand zahlreicher empirischer Studien ist zudem die Frage, ob sich der Einfluss der mütterlichen Ausbildung vom Einfluss der väterlichen Ausbildung unterscheidet (vgl. z.B. Björklund \& Salvanes, 2010; Holmlund, Lindahl, \& Plug, 
$2011)^{5}$. Die Ergebnisse dieser Studien sind jedoch sehr unterschiedlich hinsichtlich der Frage ob es überhaupt einen systematischen Unterschied gibt und falls ja ob sich dieser auf Söhne und Töchter in unterschiedlichem Masse auswirkt. Wir vermuten deshalb, dass es keinen systematischen Zusammenhang gibt. Die zweite zu testende Hypothese lautet entsprechend:

Hypothese 2: Der Einfluss des Ausbildungsabschlusses der Mutter unterscheidet sich nicht vom Einfluss des Ausbildungsabschlusses des Vaters.

\subsection{Sprachregion und Kohorteneffekte}

Die verschiedenen Sprachregionen der Schweiz weisen auch deutliche Unterschiede hinsichtlich der Bildungsmuster auf. So spielt die berufliche Bildung in der Deutschschweiz eine erheblich grössere Rolle als in der Westschweiz. ${ }^{6}$ Somit ist zu erwarten, dass in der Deutschschweiz rein berufliche Bildungspfade vergleichsweise häufiger und rein akademische Bildungspfade vergleichswese weniger häufig anzutreffen sind als in den anderen Sprachregionen. Da sowohl auf dem beruflichen als auch auf dem akademischen Bildungszweig qualitativ hochwertige Ausbildungsmöglichkeiten auf sekundärer und tertiärer Stufe angeboten werden, sind jedoch hinsichtlich der gemischten Bildungspfade keine Unterschiede zu erwarten. Die dritte Hypothese lautet wie folgt:

Hypothese 3a: In der Deutschschweiz sind rein berufliche Bildungspfade vergleichsweise häufiger und rein akademische Bildungspfade vergleichsweise weniger häufig anzutreffen als in anderen Regionen der Schweiz.

Hypothese 3b: Betreffend den gemischten Bildungspfaden bestehen keine systematischen regionalen Unterschiede.

Aufgrund der jüngeren Bildungsreformen Berufsmaturität (BMS) sowie der Einführung der Passerelle ,Berufsmaturität - Universitäre Hochschulen' sind zudem Kohorteneffekte zu erwarten. Die Berufsmaturität wurde 1993 eingeführt und

5 Es gilt zu beachten, dass sich diverse Literaturstränge mit dem Einfluss der mütterlichen im Vergleich zur väterlichen Ausbildung beschäftigen. Aufgrund des begrenzten Platzes fokussieren wir im vorliegenden Beitrag auf Studien aus der ökonomischen Literatur.

6 Siehe dazu SKBF 2014. 
kann in sechs verschiedenen Richtungen abgeschlossen werden, wobei allerdings die Grundlagenfächer aller Richtungen dieselben sind (BBT, 2012, S. 3). Mit einer Berufsmaturität können die Jugendlichen ihre Erfahrungen auf dem Arbeitsmarkt mit vertieften Kenntnissen im Beruf ergänzen und somit die Attraktivität ihrer Fähigkeiten auf dem Arbeitsmarkt deutlich steigern. Zudem erlaubt ihnen ein erfolgreicher Abschluss der Berufsmaturität eine Fachhochschule zu besuchen. Mit dem Ziel der Förderung der Durchlässigkeit des schweizerischen Bildungssystems sowie der Flexibilisierung zwischen beruflichen und akademischen Bildungsgängen wurde 2005 zudem die Passerelle Berufsmaturität eingeführt: Den Absolventen der Berufsmaturität steht seit 2005 offen eine Ergänzungsprüfung abzuschliessen, um auf alle schweizerischen Universitäten und Eidgenössischen Technischen Hochschulen zugelassen zu werden (Grob et al., 2007). Die vierte zu testende Hypothese lautet deshalb wie folgt:

Hypothese 4: Personen mit gemischten Bildungspfaden sind in jüngeren Kohorten stärker vertreten als in älteren Kohorten.

\section{Daten und empirisches Vorgehen}

Im folgenden Kapitel beschreiben wir zunächst den Datensatz Mikrozensus Ausund Weiterbildung 2011 (MZB, 2011), welchen wir für unsere empirische Analyse verwenden. In einem nächsten Schritt beschreiben wir das schweizerische Bildungssystem und erläutern, wie wir die Bildungspfade der zu untersuchenden Personen, sowie die Klassifikation der Ausbildung ihrer Eltern definieren. Zum Schluss gehen wir kurz auf die Schätzmethode ein, die wir für die vorliegende Analyse verwenden.

\subsection{Mikrozensus Aus- und Weiterbildung}

Beim Mikrozensus Aus- und Weiterbildung 2011 handelt es sich um eine Stichprobenerhebung des Bundesamtes für Statistik, die Bestandteil der Schweizer Volkszählung ist. Die Erhebung wurde mittels computergestützten telefonischen Interviews (CATI) durchgeführt. Befragt wurden Personen, die zwischen 15 und 74 Jahren alt sind und ihren Hauptwohnsitz in der Schweiz haben. Die Erhebung ist repräsentativ und enthält Informationen zu mehr als 13‘000 Personen. Diese Informationen beinhalten - neben soziodemographischen Eckdaten zu Alter, Geschlecht, beruflichem Status, etc. - detaillierte Angaben bezüglich der Bildungs- 
laufbahn. Hierzu werden für jede Person alle Ausbildungsschritte dokumentiert (Bildungsstufe, Bildungstyp und Abschlussjahr). Dadurch können komplette individuelle Ausbildungspfade rekonstruiert werden, was für unsere Studie wesentlich ist.

Für unsere empirische Analyse fokussieren wir auf Personen, die eine Ausbildung auf tertiärer Stufe aufweisen und zwischen 25 und 65 Jahren alt sind. Es ist davon auszugehen, dass eine bedeutende Anzahl von Personen unter 25 Jahren ihren Bildungspfad noch nicht abgeschlossen hat (was durch die Daten ebenfalls bestätigt wird). Für Personen über 25 Jahren berücksichtigen wir zudem, ob sie sich in einer laufenden formellen Ausbildung auf tertiärer Stufe befinden. Die obere Altersgrenze entspricht dem gesetzlichen Rentenalter für Männer im Jahr 2014. Aufgrund der begrenzten Vergleichbarkeit werden zudem Personen mit abgeschlossener Fachmittelschule ${ }^{7}$ und Lehrerausbildung aus unserer Analyse ausgeschlossen. Wir erhalten daraus eine Stichprobe mit 2322 Personen, für die wir Angaben zu allen für uns relevanten Variablen haben.

\subsection{Definition Bildungspfade}

Grundsätzlich ist das schweizerische Bildungssystem auf die Kombination von ausschliesslich beruflichen oder ausschliesslich akademischen Ausbildungen ausgerichtet. ${ }^{8}$ Rein berufliche Bildungspfade umfassen eine berufliche Grundbildung sowie anschliessend den Abschluss einer Fachhochschule, Fachschule, eidgenössischen Berufsprüfung oder höheren Fachprüfung. Somit stehen auf tertiärer beruflicher Stufe eine Vielzahl an Bildungsmöglichkeiten zur Verfügung, die den Arbeitskräften die Qualifikationen zur Ausübung anspruchsvollerer und verantwortungsvollerer Berufstätigkeiten vermitteln (SKBF, 2010, S. 244). Die tertiäre berufliche Ausbildung dient damit der Spezialisierung und der Kaderausbildung von Berufsleuten (Schweizerischer Bildungsserver, 2014). Das entscheidende Zu-

7 Fachmittelschulen bieten auf Sekundarstufe II eine dreijährige Vollzeitausbildung an. Zum Einen vermittelt diese eine vertiefte Allgemeinbildung; zum Anderen bieten sie berufsbezogene Fächer an, die auf die Berufsfelder soziale Arbeit, angewandte Psychologie und Pädagogik, Kommunikation und Information, Musik und Theater, sowie Gestaltung und Kunst vorbereiten. Fachmittelschulen bieten Zugang zu bestimmten höheren Fachschulen. Mit zusätzlicher ergänzender Allgemeinbildung erlauben sie zudem Zugang zu pädagogischen Hochschulstudiengängen, mit Fachmaturitätszeugnis Zugang zu bestimmten Fachhochschulgängen (SKBF 2006, S. 107).

8 Die folgenden Angaben basieren auf SKBF 2006, 2010, 2014, EDK, 1995, dem Schweizerischen Bildungsserver 2011 (www.educa.ch) und Hoeckel et al. 2009. 
lassungskriterium zu den genannten tertiären beruflichen Ausbildungen stellt die berufliche Grundbildung dar, wobei für Fachhochschulen zusätzlich eine eidgenössische Berufsmaturität erforderlich ist.

Rein akademische Bildungspfade umfassen eine gymnasiale Maturität sowie anschliessend den Abschluss einer Universitären Hochschule oder der Eidgenössisch Technischen Hochschulen (ETH). Der Eintritt in die Maturitätsschule ist abhängig von Erfahrungsnoten, Empfehlung der Lehrperson und bestandener Aufnahmeprüfung, wobei nicht alle Zulassungskriterien in allen Kantonen zur Anwendung kommen. Akademische Bildung auf Sekundarstufe II, d.h. die gymnasiale Maturität, vermittelt eine ausgewogene, breit gefächerte und kohärente Ausbildung, die nicht beruflich oder fachspezifisch ist, und bereitet auf den Eintritt in Universitäre, Pädagogische und Eidgenössische Technische Hochschulen vor. Akademische Bildung auf der tertiären Stufe, an einer Universitären oder einer Eidgenössischen Technischen Hochschule, vermittelt eine wissenschaftszentrierte Ausbildung und beinhaltet vorwiegend Grundlagenforschung.

Neben diesen rein akademische bzw. rein berufliche Ausbildungen umfassenden Bildungspfaden sind aufgrund der Durchlässigkeit des schweizerischen Bildungssystems jedoch auch Kombinationen von akademischen und beruflichen Ausbildungen möglich. Wir bezeichnen Bildungspfade, die sowohl akademische als auch berufliche Ausbildungen umfassen, als gemischte Bildungspfade. Auf der einen Seite sind Personen mit gymnasialer Maturität zu den beruflichen tertiären Ausbildungen zugelassen, sofern diese Personen ein gewisses Mass an beruflicher Praxis vorweisen. Auf der anderen Seite haben Personen, die die Berufsmaturität und die Ergänzungsprüfung Passerelle ,Berufsmaturität - universitäre Hochschule' absolviert haben, sowie Personen mit einem Bachelorabschluss einer Fachhochschule bei entsprechender Eignung einen grundsätzlichen Zugang zu allen universitären Hochschulen. In der vorliegenden Arbeit werden dabei alle Bildungspfade, die irgendwann einen Wechsel zwischen den beiden Bildungszweigen umfassen, unter den gemischten Bildungspfaden zusammengefasst. Inwiefern der Zeitpunkt des Wechsels (vor oder nach der tertiären Ausbildung) relevant ist, kann im Rahmen der vorliegenden Studie nicht beantwortet werden, da die Fallzahlen hierfür zu gering sind. Personen, die im Laufe ihrer Ausbildung mehrmals zwischen dem beruflichen und akademischen Bildungszweig wechseln, werden nicht berücksichtigt - wobei dies in der Schweiz (im Gegensatz zu Deutschland) kaum beobachtet wird (Bellmann \& Stephani, 2012; Hillmert \& Jacob, 2003, S. 320; Pilz, 2009).

In Abbildung 2 sind die Häufigkeiten der verschiedenen Bildungspfade abgebildet. Wie zu erwarten war, bilden Bildungspfade mit rein beruflichen, bzw. rein akademischen Ausbildungsinhalten die Mehrheit. So weisen 48\% der Personen 
mit tertiärem Abschluss einen rein beruflichen und 34\% der Personen mit tertiärem Abschluss einen rein akademischen Bildungspfad auf. Allerdings sind auch gemischte Bildungspfade keine Seltenheit. So kombinieren $18 \%$ aller Personen in unserer Stichprobe berufliche und akademische Ausbildungen. ${ }^{9}$

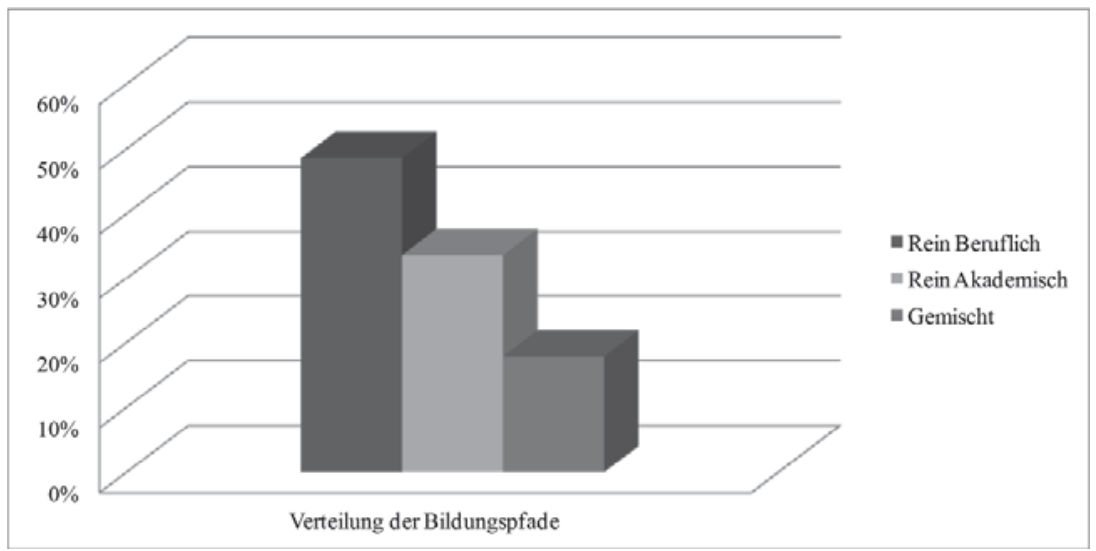

Abbildung 2 Verteilung rein akademische, rein berufliche und gemischte Bildungspfade in der Schweiz. Quelle: Eigene Berechnung, basierend auf MZB 2011.

\subsection{Definition Ausbildungsabschluss Eltern und der weiteren verwendeten Variablen}

In Bezug auf den Ausbildungsabschluss der Eltern enthält der Datensatz Informationen zur höchsten abgeschlossenen Ausbildung (nicht aber zum Bildungspfad). Daraus bilden wir eine Variable (Ausbildung Eltern), die drei Kategorien aufweist: In der ersten Kategorie sind Personen, deren Eltern keinen tertiären Ausbildungsabschluss aufweisen (kein tertiärer Abschluss). In der zweiten Kategorie sind Personen, deren Eltern keinen akademischen, mindestens ein Elternteil aber einen beruflichen Bildungsabschluss auf tertiärer Stufe aufweist (beruflicher ter-

9 Drei von vier Personen mit gemischtem Pfad starten mit akademischer Bildung, d.h. mit einer Maturität, und wechseln dann in den beruflichen Zweig. Ebenso findet der Wechsel vom akademischen zum beruflichen, bzw. vom beruflichen zum akademischen Zweig bei drei von vier Personen mit gemischtem Pfad direkt nach der Sekundarstufe II statt. 
tiärer Abschluss). In der dritten Kategorie sind Personen, von denen mindestens ein Elternteil einen akademischen Abschluss aufweist (akademischer tertiärer Abschluss). Aufgrund der bisherigen empirischen Erkenntnisse zu den Einflüssen der Ausbildung der Eltern unterscheiden wir in einem zweiten Schritt zwischen der Ausbildung des Vaters und der Ausbildung der Mutter um zu analysieren, ob diese auch für unsere Fragestellung unterschiedliche Effekte aufweisen. Dazu generieren wir zwei Variablen (Ausbildung Mutter/Vater), welche je drei Kategorien enthalten. In der ersten Kategorie der Variable Ausbildung Mutter sind Personen, deren Mutter keinen tertiären Ausbildungsabschluss aufweist (Mutter kein tertiärer Abschluss). In der zweiten Kategorie sind Personen, deren Mutter einen beruflichen Abschluss auf tertiärer Stufe hat (Mutter beruflicher tertiärer Abschluss). Die dritte Kategorie beinhaltet Personen, welche eine Mutter mit akademischem Abschluss auf tertiärer Stufe haben (Mutter akademischer tertiärer Abschluss). In Analogie dazu wird die Variable Ausbildung Vater gebildet.

In allen Schätzungen fügen wir zudem Kontrollvariablen für die Sprachregion (Linguistische Region) und für die Alterskohorte (Kohorte) ein. Bezüglich Sprachregion unterscheiden wir zwischen Deutsch, Französisch, Italienisch und Rätoromanisch (Deutsch, Französisch, Italienisch, Rätoromanisch)..$^{10}$ Bezüglich Alterskohorten bilden wir Altersgruppen im Abstand von jeweils fünf Jahren. Personen zwischen 60 und 64 Jahren sind in der ersten Kohorte (1. Kohorte), Personen zwischen 55 und 59 Jahren in der zweiten Kohorte (2. Kohorte), Personen zwischen 50 und 54 Jahren in der dritten Kohorte (3. Kohorte), etc. Tabelle 1 beschreibt die Stichprobe bezüglich rein beruflicher, rein akademischer und gemischter Bildungspfade.

10 MZB 2011 weist bilinguale Regionen nicht aus. Die Kategorisierung wiederspiegelt jene Sprache, die von der Mehrheit der Bevölkerung in einer Region gesprochen wird. 
Tabelle 1 Beschreibung der Stichprobe. Quelle: Eigene Berechnung, basierend auf MZB 2011.

\begin{tabular}{lccc}
\hline & $\begin{array}{l}\text { Rein } \\
\text { beruflich } \\
(\mathrm{N}=1023)\end{array}$ & $\begin{array}{l}\text { Rein aka- } \\
\text { demisch } \\
(\mathrm{N}=816)\end{array}$ & $\begin{array}{c}\text { Gemischt } \\
(\mathrm{N}=483)\end{array}$ \\
\hline Variable & Anteile & Anteile & Anteile \\
\hline Tertiäre Ausbildung der Mutter & & & \\
\hline Keine & $95.31 \%$ & $74.02 \%$ & $82.40 \%$ \\
Berufliche, keine akademische & $2.93 \%$ & $6.74 \%$ & $5.38 \%$ \\
Akademische & $1.76 \%$ & $19.24 \%$ & $12.22 \%$ \\
\hline Tertiäre Ausbildung des Vaters & & & \\
\hline Keine & $76.93 \%$ & $50.37 \%$ & $60.04 \%$ \\
Berufliche, keine akademische & $15.93 \%$ & $12.38 \%$ & $15.53 \%$ \\
Akademische & $7.14 \%$ & $37.25 \%$ & $24.43 \%$ \\
\hline Kohorte & & & $15 \%$ \\
\hline $64-60$ & $11.73 \%$ & $8.82 \%$ & $6.21 \%$ \\
$59-55$ & $9.29 \%$ & $7.48 \%$ & $6.63 \%$ \\
$54-50$ & $13.78 \%$ & $8.70 \%$ & $9.94 \%$ \\
$49-45$ & $14.86 \%$ & $12.99 \%$ & $14.91 \%$ \\
$44-40$ & $15.74 \%$ & $16.55 \%$ & $15.94 \%$ \\
$39-35$ & $12.81 \%$ & $12.50 \%$ & $15.53 \%$ \\
$34-30$ & $12.41 \%$ & $16.91 \%$ & $15.73 \%$ \\
$29-25$ & $9.38 \%$ & $16.05 \%$ & $15.1 \%$ \\
\hline Linguistische Region & & & \\
\hline Deutsch & $69.79 \%$ & $47.79 \%$ & \\
Französisch & $23.95 \%$ & $43.63 \%$ & \\
Italiensch & & $8.46 \%$ & \\
\hline Rätoromanisch & & $0.12 \%$ & \\
\hline
\end{tabular}




\subsection{Empirische Schätzmethode}

In einem ersten Schritt analysieren wir den Einfluss des Ausbildungsabschlusses der Eltern auf den Bildungspfad und testen Hypothesen H1 und H2. Da mit dem beruflichen, akademischen und gemischten Bildungspfad drei verschiedene Alternativen zur Verfügung stehen, verwenden wir eine multinomiale logistische Regression. ${ }^{11}$ Mit diesem Verfahren lässt sich die Wahrscheinlichkeit, einen bestimmten Bildungspfad in Abhängigkeit des Ausbildungsabschlusses der Eltern zu wählen, analysieren. Die zu erklärende Variable ist also der Bildungspfad (beruflich, akademisch oder gemischt). Als interessierende erklärende Variablen fügen wir in der ersten Schätzspezifikation die Variable Ausbildung Eltern (zur Überprüfung von H1) und in der zweiten Schätzspezifikation die zwei Variablen Ausbildung Vater/Mutter (zur Überprüfung von H2) ein. ${ }^{12}$ Zur besseren Verständlichkeit der Resultate berechnen wir jeweils die erwartete Wahrscheinlichkeit einen bestimmten Bildungspfad einzuschlagen in Abhängigkeit des Ausbildungsabschlusses der Eltern.

In einem zweiten Schritt analysieren wir regionale Unterschiede und Kohorteneffekte hinsichtlich der relativen Häufigkeit verschiedener Bildungspfade um Hypothesen $\mathrm{H} 3$ und $\mathrm{H} 4$ zu testen. Dazu präsentieren wir entsprechende deskriptive Statistiken.

11 Die entsprechende Gleichung für die multinomiale logistische Regression ist Folgende:

$$
\operatorname{Prob}\left(Y_{i}=j \mid \boldsymbol{x}_{\boldsymbol{i}}\right)=\frac{\exp \left(\boldsymbol{x}_{\boldsymbol{i}} \boldsymbol{\beta}_{j}\right)}{1+\sum_{j=1}^{3} \exp \left(\boldsymbol{x}_{\boldsymbol{i}} \boldsymbol{\beta}_{j}\right)}, j=1,2,3
$$

$\mathrm{Y}_{\mathrm{i}}$ ist der Bildungspfad des Kindes, wobei $\mathrm{j}=1$ den rein beruflichen, $\mathrm{j}=2$ den rein akademischen und $j=3$ den gemischten Pfad repräsentieren. $\mathbf{x}_{\mathrm{i}}$ enthält entweder die grobe oder die verfeinerte Variable für Ausbildung der Eltern, sowie Kontrollvariablen für linguistische Region und Kohorte.

12 Die erste Schätzung mit der erklärenden Variable Bildung Eltern zeigt den Effekt der beiden Elternteile als Paar. Die zweite Schätzung mit den zwei erklärenden Variable Bildung Vater/Mutter zeigt den Effekt der beiden Elternteile als Einzelpersonen. 


\section{$5 \quad$ Empirische Befunde}

\subsection{Familiäre Faktoren}

Den theoretischen Überlegungen folgend vermuten wir, dass der Ausbildungsabschluss der Eltern einen bedeutenden Einfluss auf den Bildungspfad der Kinder ausübt (H1). Die Resultate der multinomialen logistischen Regression bestätigen den signifikanten Zusammenhang zwischen dem Ausbildungsabschluss der Eltern und dem Bildungspfad. ${ }^{13}$ In Abbildung 3 sind die verschiedenen Wahrscheinlichkeiten einer Person, einen bestimmten Bildungspfad zu wählen, in Abhängigkeit des Ausbildungsabschlusses der Eltern dargestellt.

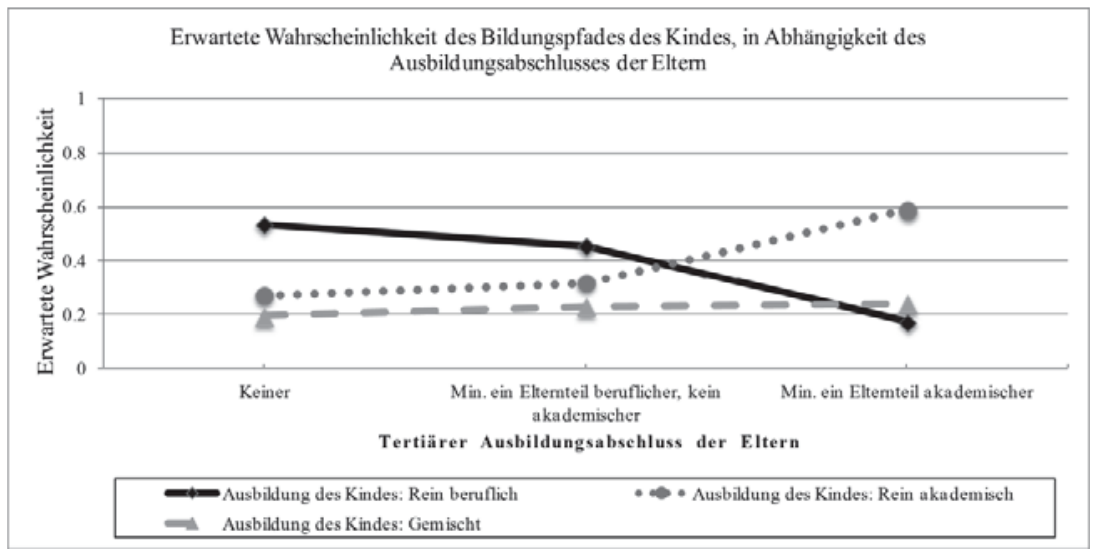

Abbildung 3 Erwartete Wahrscheinlichkeit einen bestimmten Bildungspfad einzuschlagen, in Abhängigkeit der Ausbildung der Eltern. Quelle: Eigene Berechnung, basierend auf MZB 2011.

Die Quadrate zeigen die erwartete Wahrscheinlichkeit, einen rein beruflichen Bildungspfad einzuschlagen. Personen mit Eltern ohne Ausbildung auf tertiärer Stufe haben eine Wahrscheinlichkeit von über 50\%, diesen rein beruflichen Weg einzuschlagen. Diese Wahrscheinlichkeit ist rund 10\% tiefer, wenn die Eltern einen beruflichen Abschluss auf tertiärer Stufe besitzen. Personen mit akademischen

13 Die Resultate der multinomialen logistischen Regression können auf Anfrage bei den Autoren angefordert werden. 
Eltern haben die tiefste Wahrscheinlichkeit, einen rein beruflichen Bildungspfad einzuschlagen; diese liegt bei $17 \%$.

Die Kreise zeigen die erwartete Wahrscheinlichkeit, einen rein akademischen Bildungspfad einzuschlagen. Personen, deren Eltern keinen Abschluss auf tertiärer Stufe haben, folgen diesem Bildungspfad mit einer Wahrscheinlichkeit von 27\%. Haben die Eltern einen beruflichen tertiären Abschluss, liegt sie bei 32\%. Personen mit akademischen Eltern weisen die höchste Wahrscheinlichkeit aus, diesen rein akademischen Bildungspfad zu wählen: Sie liegt bei 59\%.

Die Dreiecke zeigen die erwartete Wahrscheinlichkeit, einen gemischten Bildungspfad zu haben. Personen mit Eltern ohne Abschluss wählen einen gemischten Bildungspfad mit einer erwarteten Wahrscheinlichkeit von 19\%. Haben die Eltern einen beruflichen Abschluss, liegt diese bei 23\%. Bei Personen mit akademischen Eltern beträgt sie 24\%. Die Resultate zeigen somit, dass es keinen systematischen Zusammenhang zwischen einem bestimmten Typ eines Ausbildungsabschlusses der Eltern und gemischten Bildungspfaden der Kinder gibt.

In einem nächsten Schritt analysieren wir, ob sich der Einfluss des Ausbildungsabschlusses der Eltern zwischen Mutter und Vater unterscheidet. Unsere Hypothese lautet, dass sich der Einfluss des Ausbildungsabschlusses der Mutter nicht vom Einfluss der Ausbildungsabschlusses des Vaters unterscheidet (H2), was durch die Resultate der multinomialen logistischen Regression bestätigt wird. Die Wahrscheinlichkeiten einen bestimmten Bildungspfad zu wählen sind in Abbildung 4a und Abbildung 4b dargestellt. ${ }^{14}$

14 Die Resultate der multinomialen logistischen Regression können auf Anfrage bei den Autoren angefordert werden. 


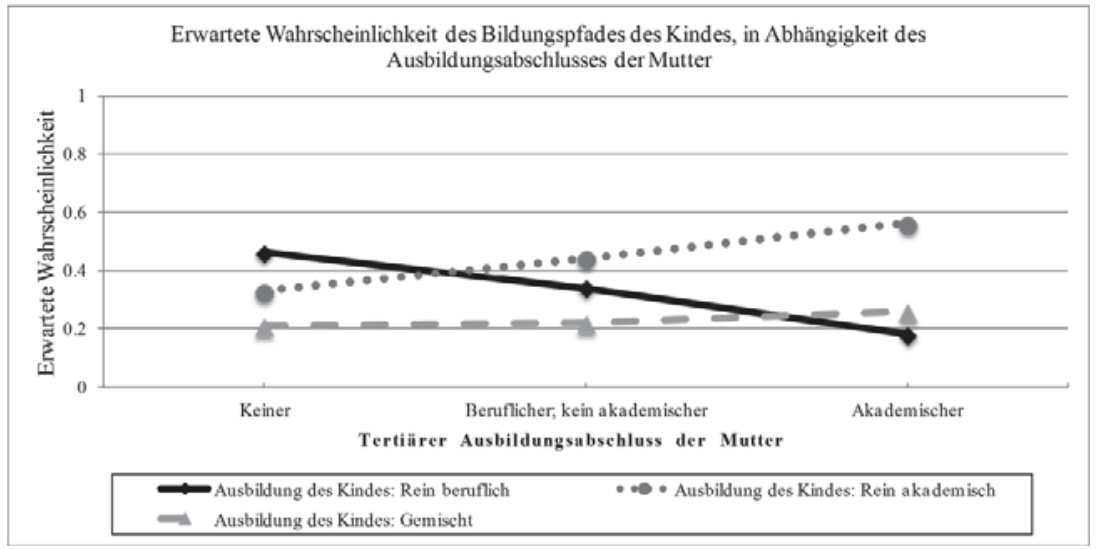

Abbildung 4a Erwartete Wahrscheinlichkeit einen bestimmten Bildungspfad einzuschlagen, in Abhängigkeit der Ausbildung der Mutter. Quelle: Eigene Berechnung, basierend auf MZB 2011.

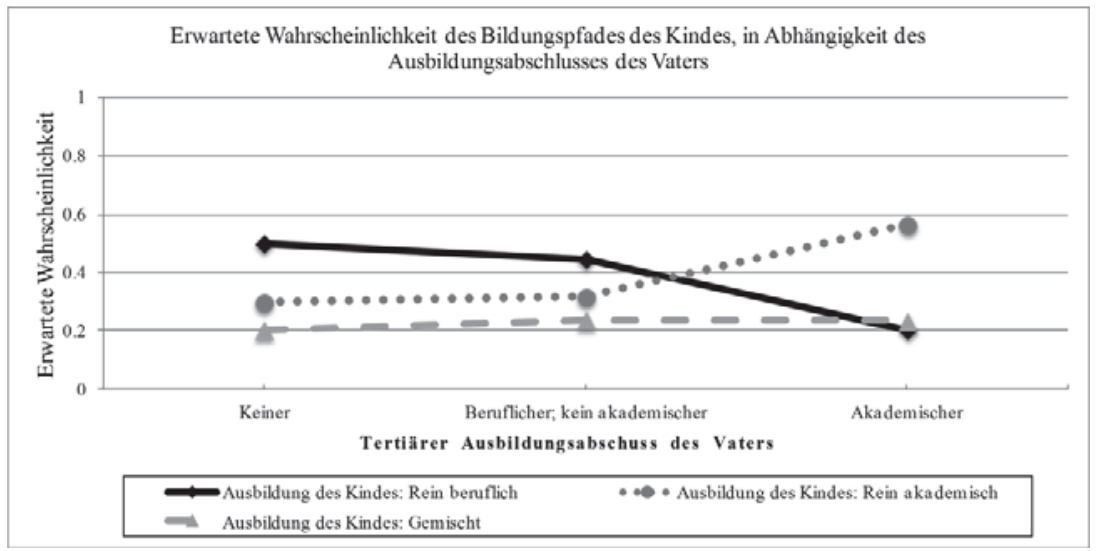

Abbildung 4b Erwartete Wahrscheinlichkeit einen bestimmten Bildungspfad einzuschlagen, in Abhängigkeit der Ausbildung des Vaters. Quelle: Eigene Berechnung, basierend auf MZB 2011. 
Die Quadrate zeigen erneut die Wahrscheinlichkeit, dass eine Person einen rein beruflichen Bildungspfad einschlägt. In Abbildung 4a ist diese in Abhängigkeit der Ausbildung der Mutter wiedergegeben, in Abbildung 4b in Abhängigkeit der Ausbildung des Vaters. Unabhängig vom Geschlecht des Elternteils ist sie am höchsten, wenn der Vater oder die Mutter keine tertiäre Ausbildung aufweisen. Die erwartete Wahrscheinlichkeit ist ebenfalls relativ hoch für Kinder, deren Mütter bzw. Väter einen beruflichen tertiären Abschluss besitzen und ist am geringsten für Kinder, deren Mütter bzw. Väter einen akademischen Abschluss aufweisen. Somit bestätigt diese separate Betrachtungsweise die bereits in Abbildung 3 aufgezeigten Zusammenhänge für den Ausbildungsabschluss der Eltern und den Bildungspfad der Kinder.

Die Kreise zeigen die Wahrscheinlichkeiten, dass sich eine Person für einen rein akademischen Bildungspfad entscheidet, wiederum separat für die Ausbildung der Mutter und die Ausbildung des Vaters. Wiederum bestätigen sich die bereits für den Ausbildungsabschluss der Eltern gefundenen Zusammenhänge: Personen, deren Mutter bzw. Vater einen akademischen Abschluss besitzen, haben die höchste Wahrscheinlichkeit einen rein akademischen Bildungspfad einzuschlagen.

Die Dreiecke zeigen die Wahrscheinlichkeiten, dass eine Person einen gemischten Bildungspfad einschlägt. Unabhängig vom Geschlecht und vom Bildungsstand der Eltern liegt diese zwischen $21 \%$ und $26 \%$ und variiert somit kaum.

Zusammenfassend zeigen die Resultate also, dass der Ausbildungsabschluss der Eltern einen signifikanten Einfluss auf den Bildungspfad der Kinder hat: Personen, deren Eltern keine tertiäre Ausbildung haben, schlagen mit höherer Wahrscheinlichkeit einen rein beruflichen Bildungspfad ein; Personen, deren Eltern ein Studium an einer Universität oder an einer ETH absolviert haben, schlagen mit höherer Wahrscheinlichkeit einen akademischen Weg ein. Hinsichtlich gemischter Bildungspfade sind keine systematischen Unterschiede in Abhängigkeit des Ausbildungsabschlusses der Eltern erkennbar. Die separaten Schätzungen für den Einfluss der Ausbildung der Mutter und der Ausbildung des Vaters bestätigen diese Ergebnisse und zeigen, dass der Einfluss der Mutter und des Vaters sich kaum unterscheidet.

\subsection{Sprachregion und Kohorteneffekte}

In einem nächsten Schritt analysieren wir regionale Unterschiede in der Verteilung der Bildungspfade. Wie erwarten, dass in der Deutschschweiz rein berufliche Bildungspfade vergleichsweise häufiger und rein akademische Bildungspfa- 
de vergleichsweise weniger häufig anzutreffen sind als in anderen Regionen der Schweiz, betreffend gemischter Bildungspfade aber keine systematischen Unterschiede bestehen (H3). Unsere deskriptiven Analysen bestätigen die Hypothese nur teilweise, da wir auch hinsichtlich der gemischten Bildungspfade systematische Unterschiede finden.

Abbildung 5 zeigt die Verteilung der rein beruflichen, der rein akademischen und der gemischten Bildungspfade innerhalb der Sprachregionen Deutsch, Französisch und Italienisch. ${ }^{15}$ Während in der Deutschschweiz über 50\% der Individuen mit tertiärer Ausbildung einen rein beruflichen Bildungspfad aufweisen, sind dies in der französischsprachigen Schweiz lediglich 32\%. Demgegenüber hat die französischsprachige Schweiz einen höheren Anteil an Personen mit rein akademischem Abschluss: Dieser beträgt über $40 \%$ in den französischsprachigen Schweiz; während in der deutschsprachigen Schweiz nur 30\% diesem Bildungspfad folgen. In der italientischsprachigen Schweiz beträgt sowohl der Anteil von Personen mit akademischer als auch beruflicher Bildung 40\%. Interessant ist nun aber der Befund, dass die relative Häufigkeit gemischter Bildungspfade in der französischsprachigen Schweiz systematisch höher ist als in den anderen Regionen: Über 25\% weisen einen Bildungspfad auf, der akademische mit beruflicher Bildung kombiniert. Die Werte in der italienisch- und der deutschsprachigen Schweiz betragen rund 15\%. Dabei sind gemischte Bildungspfade, die einen Wechsel nach einer akademischen Ausbildung auf Sekundarstufe II umfassen, am häufigsten. Somit gibt es in der französischsprachigen Schweiz einen bedeutenden Anteil von Personen, die nach einer ersten akademischen Ausbildung auf den beruflichen Bildungszweig wechseln. Ob dieser Wechsel aufgrund einer Neuorientierung oder einer Kombination komplementärer Ausbildungsinhalte geschieht, kann im Rahmen der vorliegenden Studie nicht beantwortet werden.

15 Personen, die Rätoromanisch sprechen, wurden aufgrund der Fallzahl ausgeschlossen. 


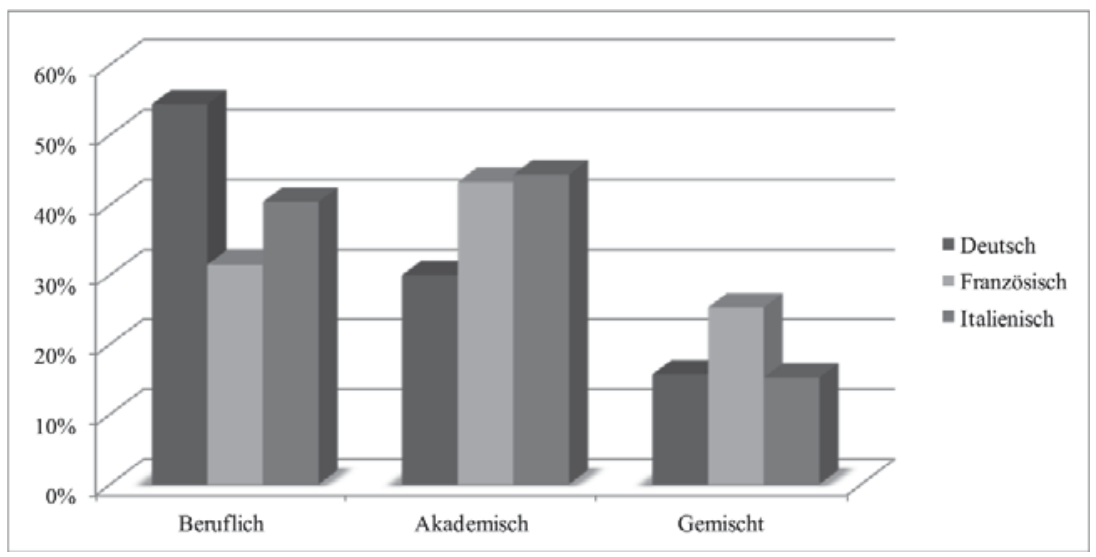

Abbildung 5 Verteilung rein akademische, rein berufliche und gemischte Bildungspfade bezüglich Sprachregionen. Quelle: Eigene Berechnung, basierend auf MZB 2011.

In einem letzten Schritt analysieren wir Kohorteneffekte. Aufgrund der jüngeren bildungspolitischen Reformen Berufsmaturität und Passerelle erwarten wir, dass berufliche und akademische Abschlüsse auf tertiärer Stufe vor allem jüngere Kohorten betreffen (H4). Aufgrund der Tatsache, dass wir in dieser Analyse auf Anteile fokussieren, haben wir - im Gegensatz zu den vorangehenden Schätzungen in dieser Analyse auch Personen mit der höchsten Ausbildung auf Sekundarstufe II einbezogen. Die empirischen Befunde dazu sind in Abbildung 6 dargestellt und bestätigen unsere Hypothese. Abbildung 6 zeigt, wie viele Personen innerhalb einer Kohorte einen rein akademischen, rein beruflichen oder gemischten Bildungspfad auf tertiärer Stufe haben, relativ zu allen Personen mit Ausbildung auf Sekundarstufe II und auf tertiärer Stufe. In der Kohorte der 60- bis 64-Jährigen haben demnach $12 \%$ einen beruflichen, $6 \%$ einen akademischen und $2 \%$ einen gemischten Bildungspfad auf tertiärer Stufe. Die restlichen $80 \%$ haben einen Abschluss auf Sekundarstufe II. 


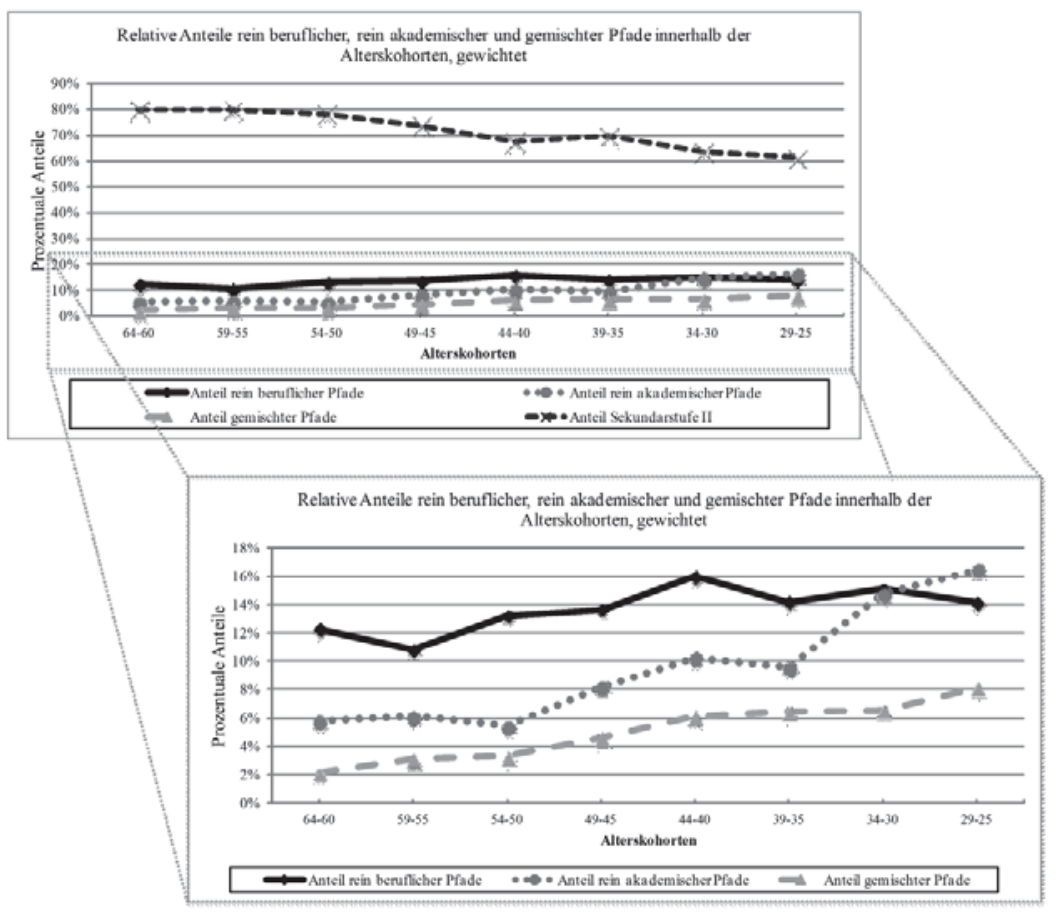

Abbildung 6 Relative Anteile rein akademischer, rein beruflicher und gemischter Bildungspfade innerhalb der Alterskohorten. Quelle: Eigene Berechnung, basierend auf MZB 2011.

Erstens fällt auf, dass ganz grundsätzlich der Anteil von tertiären Abschlüssen zugenommen hat, d.h. dass der Anteil rein beruflicher, rein akademischer und gemischter Bildungspfade in jüngeren Kohorten deutlich höher ist als in älteren Kohorten. Im Gegensatz dazu hat der Anteil von Personen, deren höchster Bildungsabschluss ein Bildungsabschluss auf Sekundarstufe II ist, abgenommen. Zweitens zeigen die Anteile rein beruflicher, rein akademischer und gemischter Bildungspfade innerhalb der tertiären Stufe, dass gemischte Bildungspfade an Bedeutung gewonnen haben, hat sich ihr Anteil in den jüngeren Kohorten doch mehr als verdoppelt. Dieses Resultat deutet darauf hin, dass die Durchlässigkeit 
des schweizerischen Bildungssystems jüngst tatsächlich erhöht wurde und auch entsprechend genutzt wird.

\section{Diskussion und Valorisierung}

In der vorliegenden Studie haben wir die Determinanten verschiedener Typen von Bildungspfaden analysiert. Dabei haben wir zwischen rein beruflichen, rein akademischen und gemischten Bildungspfaden unterschieden und den Zusammenhang zum Ausbildungsabschluss der Eltern, regionalen und zeitlichen Faktoren analysiert.

Unsere Resultate zeigen, dass zwischen dem Ausbildungsabschluss der Eltern und dem gewählten Bildungspfad ein systematischer Zusammenhang besteht. Kinder von Eltern mit einer akademischen Ausbildung wählen mit erhöhter Wahrscheinlichkeit ebenfalls einen rein akademischen Bildungspfad. Kinder von Eltern mit einer beruflichen Ausbildung wählen demgegenüber mit erhöhter Wahrscheinlichkeit einen rein beruflichen Bildungspfad. Interessanterweise findet man für gemischte Bildungspfade keinen systematischen Zusammenhang zu einem bestimmten elterlichen Ausbildungstyp. Dies spricht ebenfalls dafür, dass gemischte Bildungspfade aufgrund der Attraktivität verschiedene Ausbildungstypen zu kombinieren gewählt werden und weniger um ursprünglich eingeschlagene falsche Bildungsentscheidungen zu korrigieren.

Hinsichtlich der regionalen Verteilung zeigt sich einerseits die zu erwartende stärkere Bedeutung von beruflichen Bildungspfaden in der Deutschschweiz und akademischen Bildungspfaden in der französischsprachigen Schweiz. ${ }^{16}$ Interessanterweise sind darüber hinaus gemischte Bildungspfade in der französischsprachigen Schweiz deutlich häufiger vertreten als in den anderen Regionen. Wir vermuten, dass in der französischsprachigen Schweiz akademische Ausbildung nach wie vor eine hohe soziale Reputation geniesst und der Arbeitsmarkt aber je länger je stärker auch berufliche Ausbildungen bzw. Kombinationen von beruflichen und akademischen Ausbildungsinhalten nachfragt.

Schliesslich ist der Anteil von gemischten Bildungspfaden über die Kohorten (und in allen Regionen) angestiegen. Dies deutet darauf hin, dass die jüngeren Bildungsreformen, mit dem Ziel die Durchlässigkeit des schweizerischen Bildungssystems zu erhöhen, möglicherweise bereits greifen.

16 In der italienischsprachigen Schweiz gibt es dagegen hinsichtlich dieser beiden Bildungspfade kaum Unterschiede. 
In einem nächsten Schritt wäre es interessant weitere Determinanten (wie z.B. den Zeitpunkt der Selektion) zu analysieren. Allerdings wären für die Identifikation kausaler Effekte Paneldaten nötig, welche die Jahre der Ausbildungsentscheidungen umfassen. Solche Daten sind für die Schweiz derzeit leider (noch) nicht verfügbar. Damit könnte beispielsweise auch die potentielle Endogenität der analysierten Einflussfaktoren besser berücksichtigt werden. Diese Limitationen müssen bei der Ableitung politischer Implikationen berücksichtigt werden.

Basierend auf den Ergebnissen dieser Studie und unter Berücksichtigung früherer Studien, die die Wettbewerbsfähigkeit gemischter Bildungspfade stützen (Tuor \& Backes-Gellner, 2010 und Backes-Gellner et al., 2010) erachten wir die Erhaltung bzw. Förderung der Durchlässigkeit des schweizerischen Bildungssystems als wichtigste politische Implikation, die wir im Hinblick auf die Determinanten verschiedener Bildungspfade ableiten können. So sollen sinnvolle Kombinationen von akademischen und beruflichen Ausbildungsinhalten dadurch ermöglicht werden, dass entsprechend ausreichende Übertrittmöglichkeiten zwischen den beiden Bildungszweigen bestehen.

\section{Literaturverzeichnis}

Backes-Gellner, U., Tuor, S. N., \& Wettstein, D. (2010). Differences in the Educational Paths of Entrepreneurs and Employees. Empirical Research in Vocational Education and Training, 2(2), 83-105.

Barabasch, A., Scharnhorst, U., \& Kurz, S. (2009). Die Schweiz. In Bertelsmann Stiftung (ed.), Steuerung der beruflichen Bildung im internationalen Vergleich. (S. 241-288) Gutersloh: Bertelsmann.

Becker, G. S., \& Tomes, N. (1994). Human Capital and the Rise and Fall of Families. In G. S. Becker (Hrsg.), Human Capital. A Theoretical and Empirical Analysis with Special Reference to Education (S. 257-298). Chicago: The University of Chicago Press.

Becker, G. S. (Hrsg.). (1994). Human Capital. A Theoretical and Empirical Analysis with Special Reference to Education (3rd Edition). Chicago: The University of Chicago Press.

BBT (Bundesamt für Berufsbildung und Technologie). (2012). Schwerpunkt Berufsmaturität. Vorteile, Karrierewege und Perspektiven. FOKUS BERUF, Das Berufsbildungsmagazin für Eltern und Lehrpersonen, 3. 1-7.

Bellmann, L., \& Stephani, J. (2012). Effects of Double Qualifications on Various Dimensions of Job Satisfaction. Empirical Research in Vocational Education and Training, 4(2), 95-114.

Björklund, A., \& Salvanes, K. G. (2011). Education and Family Background: Mechanisms and Policies. In E. A. Hanushek, S. Machin, \& L. Woessmann (Hrsg.), Handbook of Economics of Education (S. 201-247). Amsterdam: Elsevier.

Breen, R., \& Jonsson, J. O. (2000). Analyzing Educational Careers. A Multinomial Transition Model. American Sociological Review, 65(5). 754-772. 
Bundesamt für Statistik (2013): Statistisches Lexikon des BFS; „Bildungsstand der Wohnbevölkerung nach Alter und Geschlecht 2013“. Stand: November 2014. Abgerufen am 17. November 2014 von http://www.bfs.admin.ch/bfs/portal/de/index/infothek/lexikon/ lex/0.topic.1.html

Connelly, R., \& Zheng, Z. (2003). Determinants of School Enrolment and Completion of 10 to 18 Year Olds in China. Economics of Education Review, 22(4). 379-388.

Culpepper, P.D. (2007), Small states and skill specificity: Austria, Switzerland and interemployer cleavages in coordinated capitalism. Comparative Political Studies, 40(6). 611-637.

Cunha, F., \& Heckman, J. J. (2007). The Technology of Skill Formation. American Economic Review, 97(2). 31-47.

Davis-Kean, P. E. (2005). The Influence of Parent Education and Family Income on Child Achievement. The Indirect Role of Parental Expectations and the Home Environment. Journal of Family Psychology, 19(2). 294-304.

Dubow, E. F., Boxer, P., \& Huesmann, R. L. (2009). Long-term Effects of Parents' Education on Children's Educational and Occupational Success. Mediation by Family Interactions, Child Aggression, and Teenage Aspirations. Merrill-Palmer Quarterly, 55(3). 224-249.

Dustmann, C. (2004). Parental Background, Secondary School Track Choice, and Wages. Oxford Economic Papers, 56(2). 209-230.

Eccles, J. S., \& Davis-Kean, P. E. (2005). Influences of Parents' Education on their Children's Educational Attainments. The Role of Parent and Child Perceptions. London Review of Education, 3(3). 191-204.

EDK (Schweizerische Konferenz der kantonalen Erziehungsdirektoren). (1995). Verordnung des Bundesrates/Reglement der EDK über die Anerkennung von gymnasialen Maturitätsausweisen (MAR). Bern: EDK.

Fan, X., \& Chen, M. (2001). Parental Involvement and Students' Academic Achievement. A Meta-Analysis. Educational Psychology Review, 13(1). 1-22.

Grob, A., Leu, E., \& Kirchhoff, E. (2007). Evaluation Passerelle. Berufsmaturität - Universitäre Hochschulen. Universität Basel.

Hanushek, E. A., Machin, S., \& Woessmann, L. (2011). Handbook in Economics of Education (Vol. 3). Amsterdam: Elsevier.

Hillmert, S., \& Jacob, M. (2003). Social Inequality in Higher Education. Is Vocational Training a Pathway Leading to or Away from University? European Sociological Review, 19(3). 319-334.

Hoeckel, K., Field, S., \& Grubb, W.N. (2009). Learning for Jobs: OECD Review of Vocational Education and Training: Switzerland. Paris: OECD.

Holmlund, H., Lindahl, M., \& Plug, E. (2011). The Causal Effect of Parents' Schooling on Children's Schooling. A Comparison of Estimation Methods. Journal of Economic Literature, 49(3). 615-651.

Leibowitz, A. (1974). Home Investment in Children. Journal of Political Economics, 82(2). 111-131.

Leibowitz, A. (1977). Parental Inputs and Children's Achievement. Journal of Human Resources, 12(2). 242-251.

Pilz, M. (2009). Why Abiturienten Do an Apprenticeship Before Going to University. The Role of 'Double Qualifications' in Germany. Oxford Review of Education, 35(2). 187-204.

Plomin, R., DeFries, J. C., \& Fulker, D. W. (1988). Nature And Nurture, During Infancy And Early Childhood. Cambridge: Cambridge University Press. 
Reynolds, A. J. (1992). Comparing Measures of Parental Involvement and Their Effects on Academic Achievement. Early Childhood Research Quarterly, 7(3). 441-462.

Rohrer, L., Trampusch, C. (2011), Continuity and change in the Swiss vocational training system. In L. Rohrer, \& C. Trampusch (Hrsg.), Switzerland in Europe: Continuity and Change in the Swiss Political Economy (S. 144-162). London: Routledge.

Sacerdote, B. (2007). How Large are the Effects from Changes in Family Environment? A Study of Korean American Adoptees. Quarterly Journal of Economics, 122(1). 119-157.

Schweizerischer Bildungsserver. (2014). Das schweizerische Bildungssystem. Stand: Dezember 2011. Abgerufen am 04. September 2007 von http://bildungssystem.educa.ch/ de/schweizerische-bildungssystem-2011

SKBF (Schweizerische Koordinationsstelle für Bildungsforschung). (2006). Bildungsbericht Schweiz: 2006. Aarau: SKBF/CSRE Schweizerische Koordinationsstelle für Bildungsforschung.

SKBF (Schweizerische Koordinationsstelle für Bildungsforschung). (2010). Bildungsbericht Schweiz: 2010. Aarau: SKBF/CSRE Schweizerische Koordinationsstelle für Bildungsforschung.

SKBF (Schweizerische Koordinationsstelle für Bildungsforschung). (2014). Bildungsbericht Schweiz: 2014. Aarau: SKBF/CSRE Schweizerische Koordinationsstelle für Bildungsforschung.

Tansel, A. (2002). Determinants of School Attainment of Boys and Girls in Turkey: Individual, Household and Community Factors. Economics of Education Review, 21(5). 455-470.

Tuor, S. N., \& Backes-Gellner, U. (2010). Risk-return Tradeoffs to Different Educational Paths: Vocational, Academic and Mixed. International Journal of Manpower, 31(5). 495519.

Turkheimer, E., Haley, A., Waldron, M., D’Onofrio, B., \& Gottesman, I. I. (2003). Socioeconomic Status Modifies Heritability of IQ in Young Children. Psychological Science, 14(6). 623-628.

Dieses Buch wird unter der Creative Commons Namensnennung-Nicht kommerziell 4.0 International Lizenz (http://creativecommons.org/licenses/by-nc/4.0/deed.de) veröffentlicht, welche für nicht kommerzielle Zwecke die Nutzung, Vervielfältigung, Bearbeitung, Verbreitung und Wiedergabe in jeglichem Medium und Format erlaubt, sofern Sie den/die ursprünglichen Autor(en) und die Quelle ordnungsgemäß nennen, einen Link zur Creative Commons Lizenz beifügen und angegeben, ob Änderungen vorgenommen wurden. 